\title{
Cardamonin, inhibits pro-inflammatory mediators in activated RAW 264.7 cells and whole blood
}

\begin{abstract}
Some chalcones, such as hydroxychalcones have been reported previously to inhibit major pro-inflammatory mediators such as nitric oxide (NO), prostaglandin E2 (PGE2), tumor necrosis factor- $\alpha$ (TNF- $\alpha$ ) and reactive oxygen species production by suppressing inducible enzyme expression via inhibition of the mitogen-activated protein kinase (MAPK) pathway and nuclear translocation of critical transcription factors. In this report, the effects of cardamonin (2',4'-dihydroxy-6'-methoxychalcone), a chalcone that we have previously isolated from Alpinia rafflesiana, was evaluated upon two cellular systems that are repeatedly used in the analysis of anti-inflammatory bioactive compounds namely RAW 264.7 cells and whole blood. Cardamonin inhibited NO and PGE2 production from lipopolysaccharide- and interferon- $\gamma$-induced RAW cells and whole blood with IC50 values of $11.4 \mu \mathrm{M}$ and $26.8 \mu \mathrm{M}$, respectively. Analysis of thromboxane B2 (TxB2) secretion from whole blood either stimulated via the COX-1 or COX-2 pathway revealed that cardamonin inhibits the generation of TxB2 via both pathways with IC50 values of 2.9 and $1.1 \mu \mathrm{M}$, respectively. Analysis of IC50 ratios determined that cardamonin was more COX-2 selective in its inhibition of TxB2 with a ratio of 0.39. Cardamonin also inhibited the generation of intracellular reactive oxygen species and secretion of TNF- $\alpha$ from RAW 264.7 cells in a dose responsive manner with IC50 values of $12.8 \mu \mathrm{M}$ and $4.6 \mu \mathrm{M}$, respectively. However, cardamonin was a moderate inhibitor of lipoxygenase activity when tested in an enzymatic assay system, in which not a single concentration tested was able to cause an inhibition of more than $50 \%$. Our results suggest that cardamonin acts upon major pro-inflammatory mediators in a similar fashion as described by previous work on other closely related synthetic hydroxychalcones and strengthens the conclusion of the importance of the methoxyl moiety substitution on the $4^{\prime}$ or $6^{\prime}$ locations of the A benzene ring.
\end{abstract}

Keyword: Cardamonin, Alpinia rafflesiana, RAW 264.7, Whole blood, Inflammatory mediator 